\title{
LAPTM4B Down Regulation Inhibits the Proliferation, Invasion and Angiogenesis of HeLa Cells In Vitro
}

\author{
Fanling Meng ${ }^{\mathrm{a}}$ Xiuwei Chen ${ }^{\mathrm{a}}$ Hongtao Song ${ }^{\mathrm{b}}$ Ge Lou $^{\mathrm{a}}$ \\ aDepartment of Gynecology, The Affiliated Tumor Hospital, Harbin Medical University, Harbin, \\ bDepartment of Pathology, The Affiliated Tumor Hospital, Harbin Medical University, Harbin, China
}

\author{
Key Words \\ LAPTM4B • Cervical cancer • RNA interference • Proliferation • Invasion • Angiogenesis
}

\begin{abstract}
Background/Aims: LAPTM4B (lysosome-associated protein transmembrane 4 beta) is a novel oncogene with important functions in aggressive human carcinomas, including cervical cancer. However, the specific functions and internal molecular mechanisms associated with this gene in the context of cervical cancer remain unclear. Methods: In this study, we explored the effects and mechanisms of LAPTM4B on tumor growth, metastasis and angiogenesis in vitro by depletion ofLAPTM4B in Hela cell. RNA interference was used to induce down regulation of LAPTM4B gene expression in Hela cells. The motility, migration potential, and proliferation of the Hela cells were measured by flow cytometry, Transwell migration assays, wound healing assays, and Cell Counting Kit- 8 assays. In addition, the cell cycle analysis utilized fluorescenceactivated cell sorting. Results: In this study, RNAi-mediated LAPTM4B knockdown inhibited cell growth and angiogenesis. In vitro, HeLa cells with down regulated LAPTM4B also exhibited decreased migration and invasion activity as well as significantly reduced CDK12, HIF-1 $\alpha$, MMP2, MMP-9 and VEGF expression. LAPTM4B blockade significantly decreased cord lengths and branch points in a tube formation assay. Conclusions: These results suggested that LAPTM4B inactivation could be a novel therapeutic target for cervical cancer.
\end{abstract}

Copyright $\odot 2015$ S. Karger AG, Basel

\section{Introduction}

Cervical cancer, which is one of the most common cancers, remains the leading cause of cancer deaths among females worldwide [1]. Approximately one-third of patients who present with invasive cervical cancer will die from this disease. More than $85 \%$ of cervical cancer cases and deaths occur in developing countries, including China, where chemotherapy is used to treat recurrent cervical cancer [2]. In China, there are approximately 130,000 


\section{Cellular Physiology Cell Physiol Biochem 2015;37:890-900 \begin{tabular}{l|l|l|l|l}
\hline DOI: 10.1159/000430216 & O 2015 S. Karger AG, Basel
\end{tabular} and Biochemistry Published online: September 18, 2015 www.karger.com/cpb}

new cervical cancer cases and 50,000 deaths from cervical cancer each year [3]. Although advanced surgical techniques and chemoradiotherapy can improve the efficacy of cervical cancer treatment, the mortality rate of this disease remains high due to tumor recurrence and drug resistance during chemoradiotherapy [4-7]. Therefore, efforts to identify additional novel molecular markers for the detection and diagnosis of cervical cancer are of great clinical importance.

Lysosome-associated protein transmembrane 4 Beta (LAPTM4B) is a specific transcription factor in the mammalian 4-tetratransmembrane spanning protein superfamily [8]. The overexpression and aberrant function of LAPTM4B have been verified in cases of carcinoma progression and in various malignant carcinomas, such as breast cancer [9], lung cancer [10], gastric cancer [11], hepatocellular carcinoma [12], prostate cancer [13] and gallbladder carcinoma [14]. Moreover, LAPTM4B is known to be a dynamic cancerassociated biomarker that is involved in cell cycle progression, differentiation, DNA damage repair, angiogenesis and other biological processes [15].

In prior research, we observed the highly frequent overexpression of the LAPTM4B protein in cervical carcinoma and noted that this overexpression was associated with unfavorable clinicopathological findings and poor survival [16]. These results suggested that LAPTM4B could be a valuable biological marker for cervical cancer. However, the roles of the LAPTM4B gene in cervical cancer and the molecular mechanisms through which this gene performs its functions remain unknown. Although LAPTM4B is known to be a candidate oncogene and/or a potential therapeutic target in various cancers, the questions of whether this gene has similar roles in cervical cancer and whether it is involved in cervical cancer tumorigenesis are of great interest. In the present study, we investigated these questions in cervical cancer cells in vitro.

\section{Materials and Methods}

Cell lines

Three human cervical cancer cell lines (SiHa, HeLa and CaSki cells) were obtained from the ATCC (Rockville, Maryland, USA). These lines were maintained in Dulbecco's modified Eagle's medium (DMEM) containing $10 \%$ fetal bovine serum (FBS).

Construction of expression plasmids and screening of target sites

Four precursor microRNA (pre-miRNA) sequences that target LAPTM4B (GenBank accession number: NM_018407.4) were designed using Invitrogen Block-iT RNAi Designer, an RNAi design software from Invitrogen Corporation. The pre-miRNA sequencing primers are presented in Fig. 1A. Double-stranded oligonucleotides encoding the pre-miRNA sequences were then ligated into the linearized pcDNA6.2GW/EmGFP-miR vector (Invitrogen) to form the recombinant plasmids pcDNA-LAPTM4B-miR-1, pcDNALAPTM4B-miR-2, pcDNA-LAPTM4B-miR-3, and pcDNA-LAPTM4B-miR-4. Subsequently, competent DH5 $\alpha$. coli cells were transformed using these plasmids. All constructs were verified by sequencing. Lipofectamine 2000 reagent (Invitrogen) was then used to transfect these plasmids into 293T cells in accordance with the manufacturer's instructions for virus packages and knockdown efficiency tests.

\section{Preparation and titering of LAPTM4B RNAi lentiviral particles}

The lentiviral expression construct pLenti6/V5-GW/Em-GFP-miR was produced by rapid BP/LB recombination reactions among pDONR TM221, pcDNA-LAPTM4B-miR3, and pLenti6/V5-DEST.The recombinant lentiviral vector and lentiviral packaging mix were co-transfected into 293T cells. Lentiviruscontaining supernatant was filtered and concentrated at $48 \mathrm{~h}$ after transfection.

Construction of the LAPTM4B miRNA lentiviral vector and cell infection

The most effective LAPTM4B RNAi lentiviral vectors, as determined by qPCR and western blotting, were inserted into pLenti6/V5-DEST (Invitrogen) to generate miRNA lentiviral vectors, following the manufacturer's instructions. The resulting plasmids were confirmed by sequencing and named pcDNALAPTM4B-miR and pcDNA-LAPTM4B-miR-neg. The lentiviral vectors and packaging vectors were then 


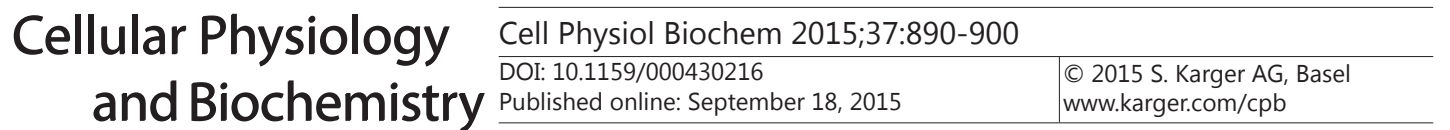

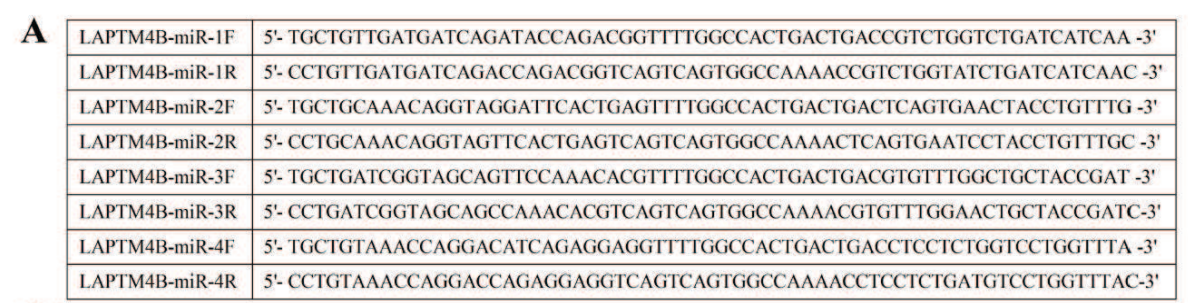

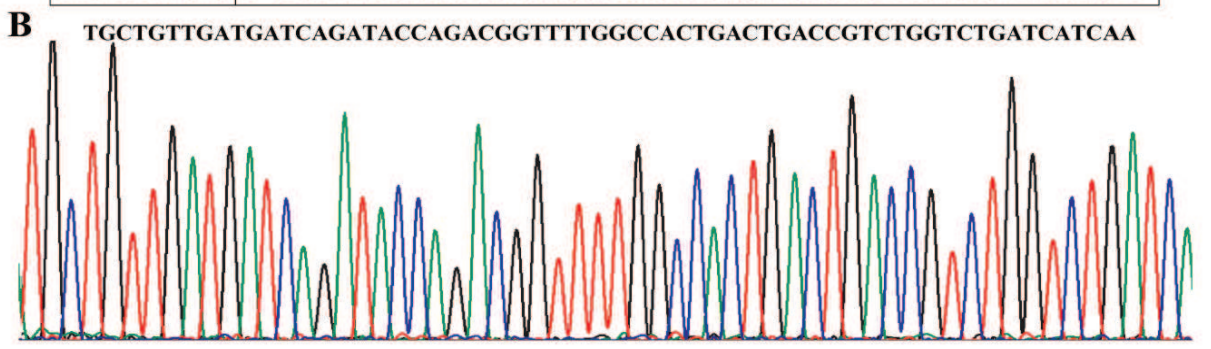

pcDNA-LAPTM4B-miR-SR1

TGCTGTTGATGATCAGATACCAGACGGTTTTGGCCACTGACTGACCGTCTGGTCTGATCATCAA

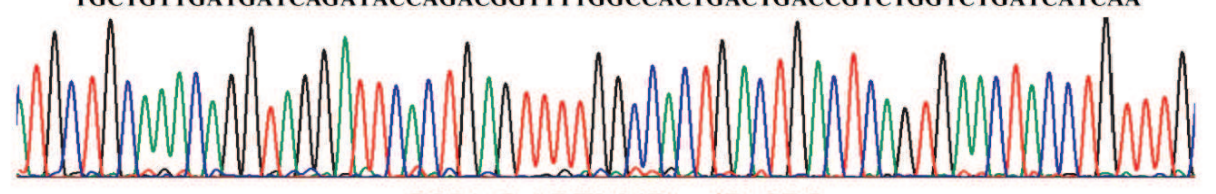

pcDNA-LAPTM4B-miR-SR2

TGCTGATCGGTAGCAGTTCCAAACACGTTTTGGCCACTGACTGACGTGTTTGGCTGCTACCGAT

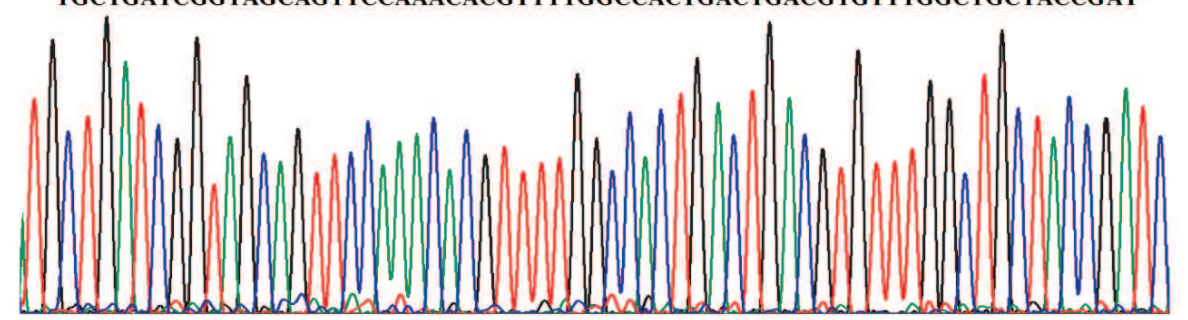

pcDNA-LAPTM4B-miR-SR3

TGCTGTAAACCAGGACATCAGAGGAGGTTTTGGCCACTGACTGACCTCCTCTGGTCCTGGTTTA

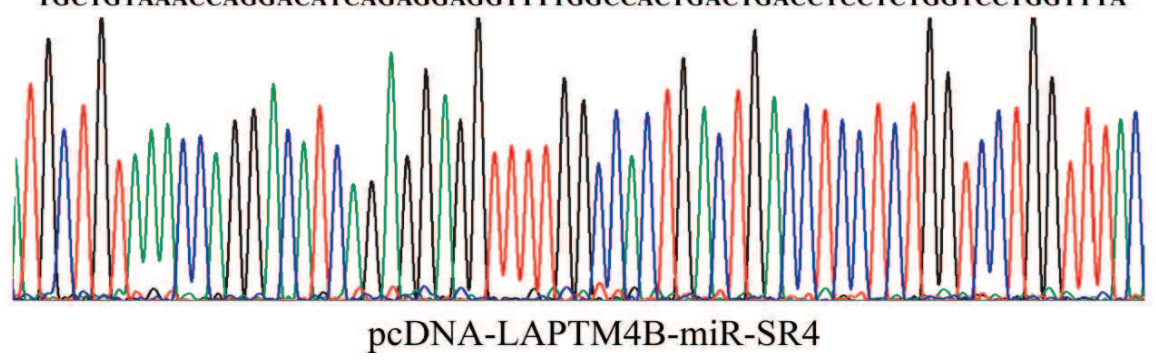

Fig. 1. Construction of recombinant plasmids containing the miRNA insert fragments against LAPTM4B. (A) Predicted secondary structure of the pre-miRNA targeting LAPTM4B (SR1, SR2, SR3, and SR4). (B) The DNA sequencing results showed that the inserts were correct, and no mutant was found in the recombinants.

transfected into 293T cells. Virus titers were determined and calibrated in these 293T cells using a green fluorescent protein (GFP) assay, and the 293T cell culture medium was centrifuged and concentrated to obtain an LAPTM4B miRNA lentiviral solution that was called lenti-LAPTM4B. Lentiviral titers were then determined by infecting $293 \mathrm{~T}$ cells. The HeLa cells were infected with lentivirus at a multiplicity of infection (MOI) of 1 in the presence of $100 \mu \mathrm{g} / \mathrm{ml}$ of polybrene. The efficiency of LAPTM4B knockdown by this infection was tested by qPCR and western blotting. The infected cells were cultured for two weeks in a selection medium containing $6 \mathrm{mg} / \mathrm{ml}$ blasticidin (Invitrogen) to produce stably expressing cell lines. After selection, $3 \mu \mathrm{g} / \mathrm{ml}$ blasticidin was used to obtain stable cell lines. 


\section{Cellular Physiology Cell Physiol Biochem 2015;37:890-900 \begin{tabular}{l|l|l}
\hline DOI: 10.1159/000430216 & O 2015 S. Karger AG, Basel
\end{tabular} and Biochemistry Published online: September 18, $2015 \quad$ www.karger.com/cpb \\ Meng et al.: LAPTM4B Inhibits Invasion and Angiogenesis of Cervical Cancer}

Real-time RT-PCR

Total RNA was extracted with TRIzol reagent (Invitrogen Life Technologies, Carlsbad, CA, USA). A Superscript III Platinum Kit (Invitrogen) was used to reverse transcribe this total RNA into cDNA. Real-time PCR was performed with SYBR Green Master Mix (TaKaRa, Kyoto, Japan), using GAPDH as an endogenous reference gene. The primer sequences were as follows: LAPTM4B forward primer, $5^{\prime}$-GTT ACC AGC AAT GAC ACT ACGG-3'; LAPTM4B reverse primer, 5'-CTC CTT GGC AGC ACC ATT-3'; GAPDH forward primer, 5'-GGG TGT GAA CCA TGA GAA GTATG-3'; and GAPDH reverse primer, 5'-GAT GGC ATG GAC TGT GGT CAT-3'. The PCR cycling conditions were $2 \mathrm{~min}$ at $95^{\circ} \mathrm{C} ; 40$ cycles of $95^{\circ} \mathrm{C}$ for $15 \mathrm{~s}$ and $59^{\circ} \mathrm{C}$ for $20 \mathrm{~s}$; and a final extension at $72^{\circ} \mathrm{C}$ for $20 \mathrm{~s}$.

\section{Western blot analysis}

Protein expression levels were evaluated by western blot analyses with specific antibodies. The proteins in each cell were extracted using PIPA buffer (Beyotime). Equal quantities of protein were subjected to $12 \%$ sodium dodecyl sulfate polyacrylamide gel electrophoresis (SDS-PAGE) and transferred topolyvinylidene difluoride (PVDF) membranes. These membranes were incubated with each primary antibody overnight at $4^{\circ} \mathrm{C}$ and then with horseradish peroxidase-conjugated secondary antibody for 2 hours at room temperature. After extensive washing, immunoreactivity was visualized using an enhanced chemiluminescence (ECL) system (ECL kit, Pierce Biotechnology). The housekeeping gene GAPDH was used as an endogenous protein for normalization.

\section{Cell proliferation assay}

The cell proliferation assay was performed with Cell Counting Kit-8 (CCK-8; Dojindo, Kumamoto, Japan). Cells were seeded into 96 -well plates at $1 \times 10^{4}$ cells per well and then incubated for 5 days. Each time point, $10 \mu \mathrm{l}$ of CCK-8 solution was added to each well and incubated for $2 \mathrm{~h}$ at $37^{\circ} \mathrm{C}$. Then the results were measured with a microplate reader at $450 \mathrm{~nm}$ in accordance with the manufacturer's instructions.

\section{Cell cycle assay}

HeLa cells were seeded into 6-well plates at a density of $1 \times 10^{6}$ cells per well. Subsequently, cells were harvested, fixed by suspension in $70 \%$ cold ethanol, and stored at $-20^{\circ} \mathrm{C}$ overnight. The ethanol was washed away with PBS, propidium iodide $(100 \mu \mathrm{g} / \mathrm{ml})$ solution was added for $1 \mathrm{~h}$, and the cells were treated with DNase-free RNase A solution $(0.5 \mathrm{mg} / \mathrm{ml})$ for $30 \mathrm{~min}$. The stained cells were analyzed by flow cytometry (FACSCalibur, BD Biosciences, San Diego, CA, USA) to examine their cell cycle distribution.

\section{Cell apoptosis assay}

Apoptosis was detected by flow cytometric analysis. In brief, HeLa cells at a density of $5 \times 10^{6}$ were collected, washed and resuspended in PBS containing annexin V-fluorescein isothiocyanate (FITC; $5 \mu \mathrm{l} /$ $\mathrm{ml}$ ). Subsequently, propidium iodide (Shanghai GeneChem Biotech Co., Ltd.) was added, and the cells were incubated for $20 \mathrm{~min}$ at $4^{\circ} \mathrm{C}$. Finally, cell apoptosis in the examined HeLa cells was assessed by flow cytometry.

\section{Cell invasion and migration assays}

The upper side of a polycarbonate membrane of a Transwell filter insert ( $8 \mu \mathrm{m}$; Millipore, USA) was coated with Matrigel (BD Biosciences, CA). After $30 \mathrm{~min}$ of incubation at $37^{\circ} \mathrm{C}$ in $5 \% \mathrm{CO}_{2}$, the Matrigel had solidified; it served as the extracellular matrix, forming a continuous thin layer for tumor cell invasion analyses. Cells were harvested in $100 \mu \mathrm{l}$ of serum-free medium and added to the upper compartment of the chamber. Cells that had migrated from the Matrigel into the pores of the inserted filter were fixed with $100 \%$ methanol for $30 \mathrm{~min}$. The lower chamber was then filled with $1 \%$ crystal violet for $20 \mathrm{~min}$. Finally, the cells were counted and photographed under an inverted microscope. The procedure for the migration assay was similar to the procedure for the Transwell invasion assay, although the inner surface of the chamber was not coated with Matrigel in the migration assay.

ELISA for VEGF

An ELISA was performed to determine VEGF concentrations. In brief, cultured cells were first incubated in 6-well plates for 24 hours. VEGF concentration was then measured using an ELISA kit (R\&D Systems, Inc., 


\section{Cellular Physiology Cell Physiol Biochem 2015;37:890-900 \begin{tabular}{l|l|l|}
\hline DOI: 10.1159/000430216 & O 2015 S. Karger AG, Basel
\end{tabular} and Biochemistry Published online: September 18, 2015 www.karger.com/cpb \\ Meng et al.: LAPTM4B Inhibits Invasion and Angiogenesis of Cervical Cancer}

Minneapolis, Minnesota, USA), in accordance with the manufacturer's instructions. In particular, absorbance values at $450 \mathrm{~nm}$ were determined with a microplate reader. Standard curves relating concentrations to absorbance values were then plotted.

\section{In vitro tube formation assay}

The angiogenesis in vitro assay was conducted in 96-well plates coated with $50 \mu \mathrm{l}$ of ECMatrix ${ }^{\mathrm{TM}}$ (BD Biosciences) following the manufacturer's instructions. The tube formation assay is based on the ability of endothelial cells to form 3D capillary-like tubular structures when cultured on a gel of basement membrane extract. Before testing was performed, $70 \mu \mathrm{L}$ of Matrigel Basement Membrane Matrix (BD Biosciences) was pipetted into a 24-well plate and incubated for 30 minutes at $37^{\circ} \mathrm{C}$. HUVECs were resuspended in collected supernatants from the knockdown (LAPTM4B-miR-3 cells), the blank control (the untreated Hela cells), and the negative control groups (the lenti-LAPTM4B-miR-neg cells), respectively. Human umbilical vein endothelial cells (HUVECs; $2 \times 10^{4}$ cells) were placed on a layer of polymerized Matrigel and incubated at $37^{\circ} \mathrm{C}$ for 24 hours. The lumen formation of these HUVECs was then analyzed by counting the number of connecting branches between cells. Each experiment was performed at least thrice.

\section{Statistical analysis}

The data are expressed as means \pm SD (standard deviation) where applicable. Statistical analyses of the data were performed using ANOVA, followed by post-hoc Dunnett's t-tests. $P<0.05$ was considered significant.

\section{Results}

\section{Expression of LAPTM4B in human cervical cancer cell lines}

RT-PCR analyses were used to determine LAPTM4B expression levels in three different cervical cancer cell lines: CaSki, SiHa, and HeLa cells. These three cell lines exhibited differing levels of LAPTM4B expression, with the highest LAPTM4B expression observed in the HeLa cell line (Fig. 2).

\section{Identification of specific and efficient miRNA sequences against LAPTM4B}

DNA sequencing results indicated that the inserts in the four pre-miRNA sequences that target LAPTM4B were correct (Fig. 1B, C). The successful transient transfection of the pcDNA-LAPTM4B-miR plasmids into the 293T cell line was confirmed by utilizing fluorescence microscopy to detect GFP expression. Both real-time RT-PCR and western blot assays indicated that among the four recombinant plasmids, pcDNA-LAPTM4B-miR3 was most efficient at knocking down LAPTM4B (Fig. 3). Therefore, the pcDNA-LAPTM4B-miR3 vector was inserted into the lentiviral vector for LAPTM4B RNAi.

Fig. 2. Expression levels of LAPTM4B in cervical cancer cells. Gene expression levels of LAPTM4B in ovarian cancer cells analyzed by real-time PCR.

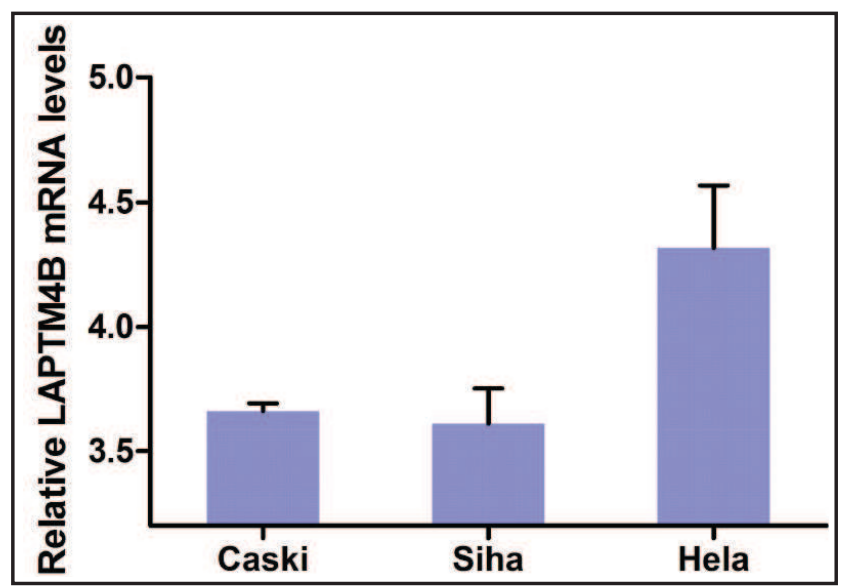




\section{Cellular Physiology Cell Physiol Biochem 2015;37:890-900 \begin{tabular}{l|l} 
DOI: 10.1159/000430216 & \\
and Biochemistry & $\begin{array}{l}\text { P } 2015 \text { S. Karger AG, Base } \\
\text { www.karger.com/cpb }\end{array}$ \\
\cline { 2 - 3 }
\end{tabular}

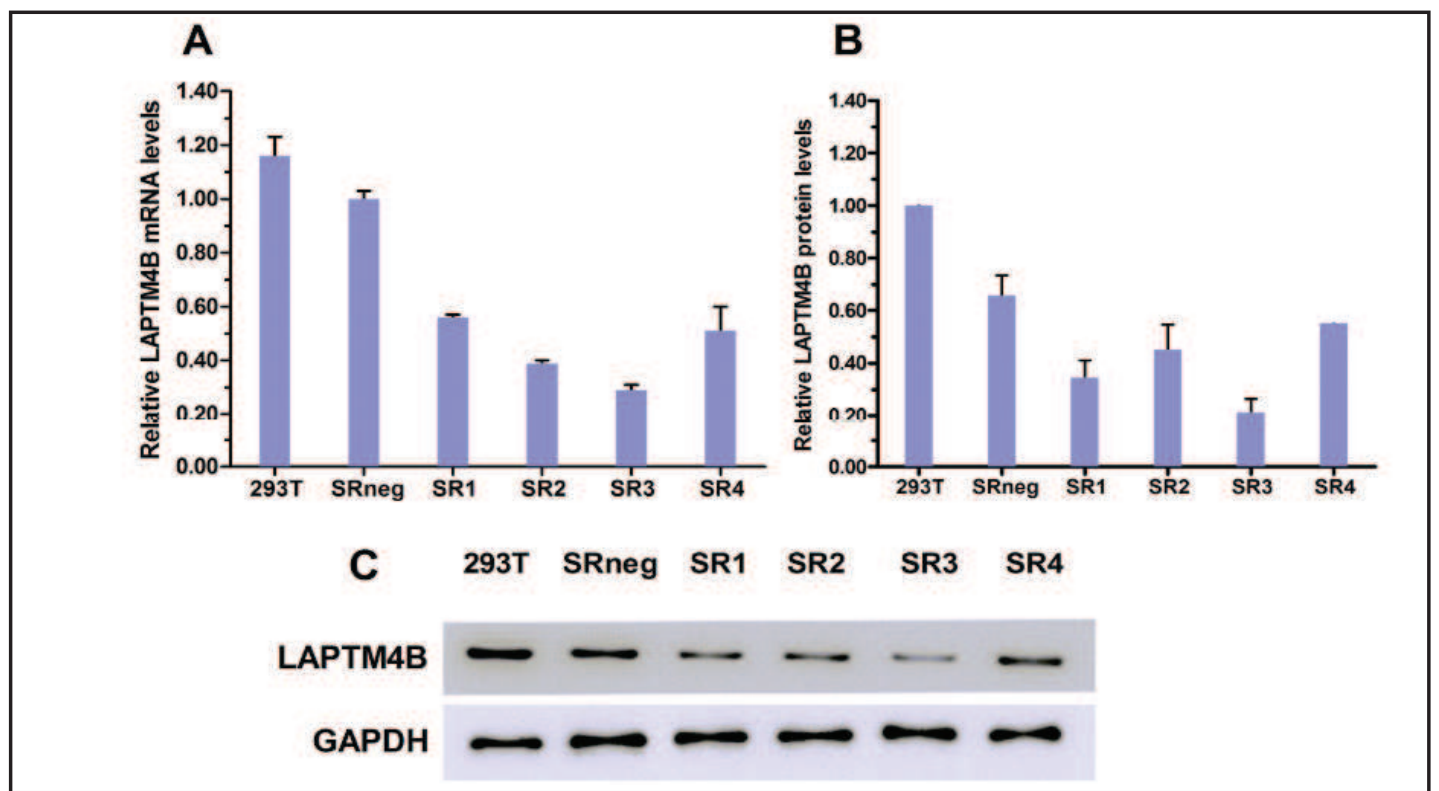

Fig. 3. Impact of recombinant plasmid expression vectors pcDNA-LAPTM4B-miRs on LAPTM4B expression levels. (A) Summary of real-timeRT-PCR results for LAPTM4B-miRNA-transfected cells. (B and C) Western blot analysis showed LAPTM4B protein was most reducedin 293T cells transfected with Lenti-LAPTM4B-SR3.
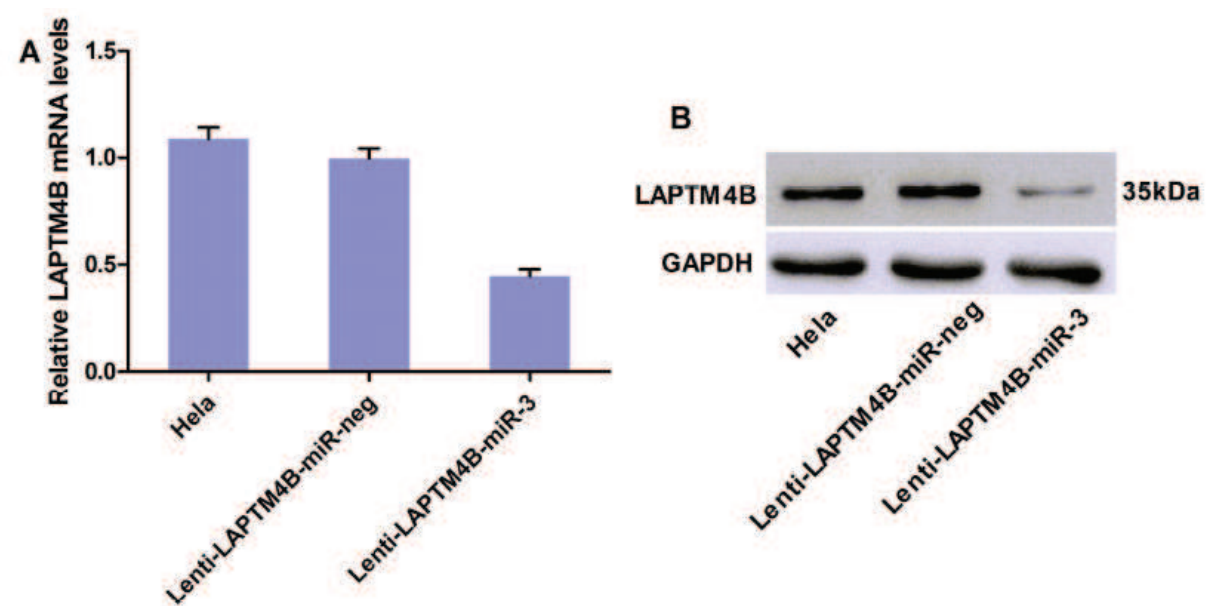

Fig. 4. Transfection of recombinant lentivirus vector into Hela cells and stable knockdown of LAPTM4B. (A) Real-time RT-PCR demonstrated the down-regulation of LAPTM4B mRNA in lenti-LAPTM4B-miR-3 cells transfected by lenti-LAPTM4B (the knockdown group) compared to the Hela cells (the control group) and lenti-LAPTM4B-miR-neg cells (the negative control group). (B) Western blot analysis showed significantly reduction of LAPTM4B protein in lenti-LAPTM4B-miR-3 cells.

\section{Propagation of stable LAPTM4B-silencing cell lines using recombinant lentivirus-mediated} MiRNA

We obtained a stable LAPTM4B-silenced cell line that we named lenti-LAPTM4B-miR-3. LAPTM4B mRNA and protein levels were significantly down regulated in lenti-LAPTM4BmiR-3 cells relative to parental HeLa cells and lenti-LAPTM4B-miR-neg cells (Fig. 4A, B).

\section{Cell proliferation determined by the CCK- 8 assay}

Cell proliferation was measured by the CCK-8 assay. We found that in the HeLa cell line, cells subjected to LAPTM4B knockdown exhibited decreased proliferation relative to untreated cells and cells infected with the Lenti-NC virus (Fig. 5A).

\section{KARGER}




\section{Cellular Physiology Cell Physiol Biochem 2015;37:890-900 \begin{tabular}{l|l|l} 
DOI 10.1159/000430216 & $\begin{array}{l}\text { O 2015 S. Karger AG, Base } \\
\text { www.karger.com/cpb }\end{array}$ \\
\cline { 2 - 3 }
\end{tabular}
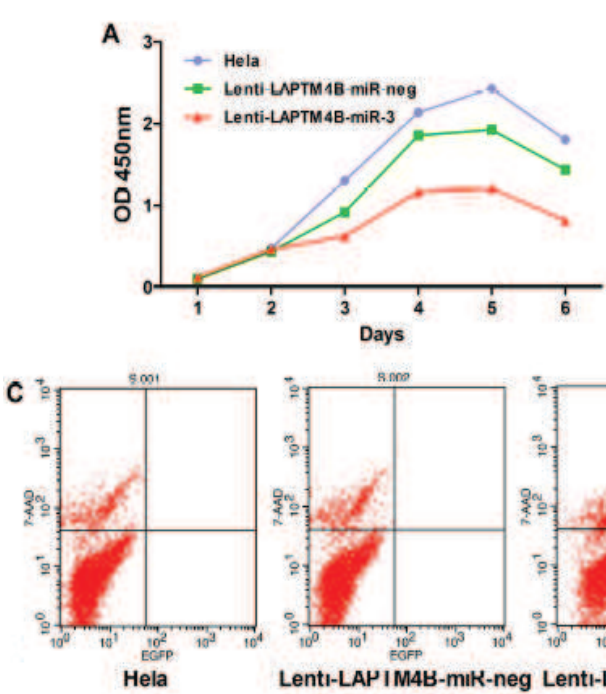

LentI-LAPIM4B-miK-neg

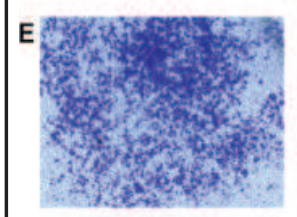

Hela

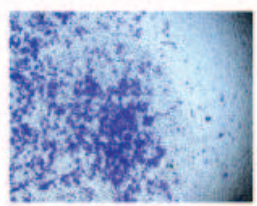

Lenti-LAPTM4B-miR-neg Lenti-LAPTM4B-miR-3

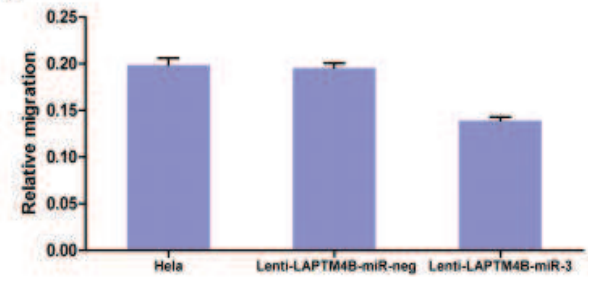

D
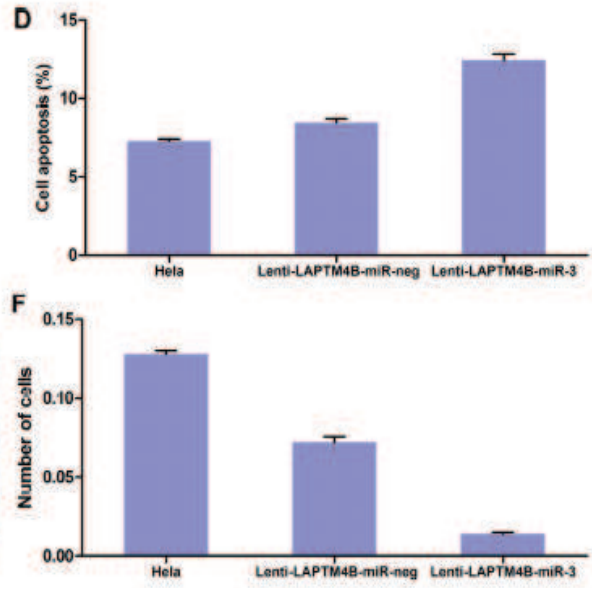

Fig. 5. Knockdown of LAPTM4B gene inhibited the proliferation, migration and invasion of Hela cells. (A) CCK8 assay showed that the cell proliferation of lenti-LAPTM4B-miR-3 was decreased compared with the Hela and lenti-LAPTM4B-miR-neg cells. (B) Migration assays were used to evaluate the migration of Hela, lenti-LAPTM4B-miR-neg and lenti-LAPTM4B-miR-3 cells. (C and D) Quantitative analysis of cell apoptosis upon LAPTM4B silencing by Annexin V and PI double staining. Data were Mean \pm SE for triplicate experiments $(\mathrm{P}<0.05)$. (E) Transwell invasion assays are demonstrated for each group. (F) Histogram analysis of the number of transmembrane cells. The data are presented as mean \pm SD from triplicates $(P<0.05)$.

Cell cycle regulation and apoptosis as measured by flow cytometry

The effects of LAPTM4B knockdown on HeLa cell cycle progression and apoptosis were analyzed by flow cytometry. Fluorescence-activated cell sorting (FACS) results indicated that LAPTM4B knockdown did not significantly affect the cell cycle (data not shown). However, cells subjected to LAPTM4B knockdown exhibited significantly increased apoptosis relative to untreated cells. These data suggest that LAPTM4B knockdown promotes apoptosis in HeLa cells (Fig. 5C, D).

Transwell invasion and migration assay

The study results indicated that LAPTM4B knockdown may suppress HeLa cell migration in vitro (Fig. 5B). Subsequently, the ability to invade Matrigel was assessed using a Transwell assay. Images of cells stained with crystal violet suggested that the knockdown of LAPTM4Bexpression significantly reduced quantities of invaded cells $(P<0.05$, Fig. 5E, F).In combination, these data indicate that LAPTM4B is involved in the migration and invasion of cervical cancer cells.

Expression of tumor-associated proteins

Several proteins that perform primary functions in the invasion, migration and metastasis of cervical cancer include VEGF, MMP-2, MMP-9, CDK12 and HIF- $1 \alpha$.The western blot analysis results demonstrated thatLAPTM4B down regulation decreased VEGF, MMP-2, MMP-9, CDK12 and HIF-1 $\alpha$ expression levels (Fig. 6). 


\section{\begin{tabular}{lll} 
Cellular Physiology & \multicolumn{1}{c}{ Cell Physiol Biochem 2015;37:890-900 } & \\
\cline { 2 - 3 } DOI: 10.1159/000430216 & O 2015 S. Karger AG, Basel
\end{tabular} and Biochemistry Published online: September 18, $2015 \quad$ www.karger.com/cpb}

Fig. 6. Expression levels of some tumor-associated proteins of Hela cells after knockdown of LAPTM4B determined by western blotting.

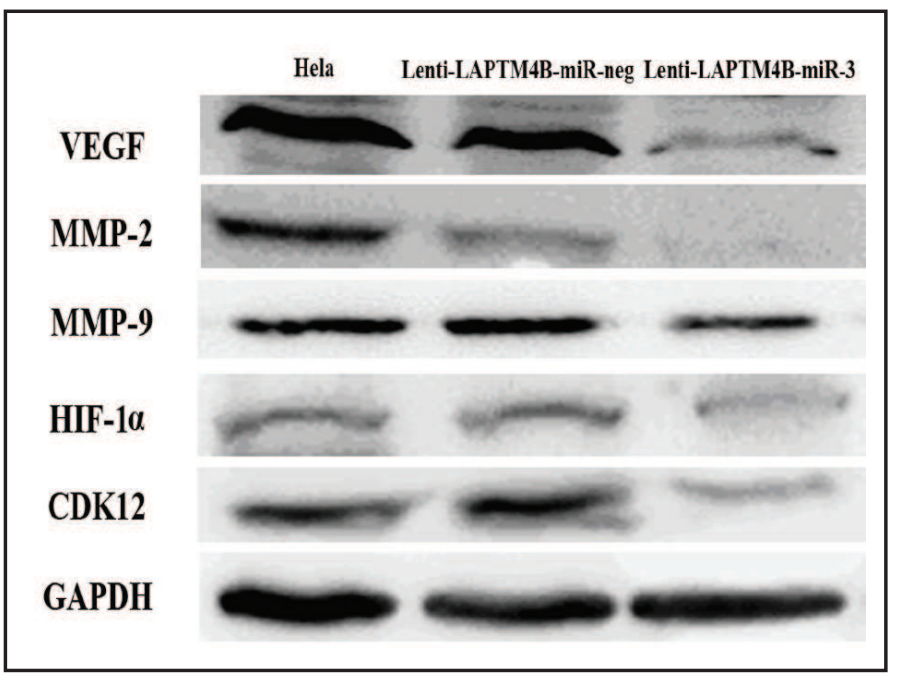

A

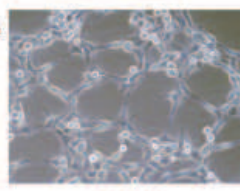

Hela

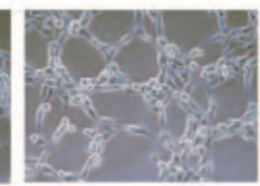

Lenti-LAPTM4B-miR-neg Lenti-LAPTM4B-miR-3

$\mathrm{C}_{1.5}$

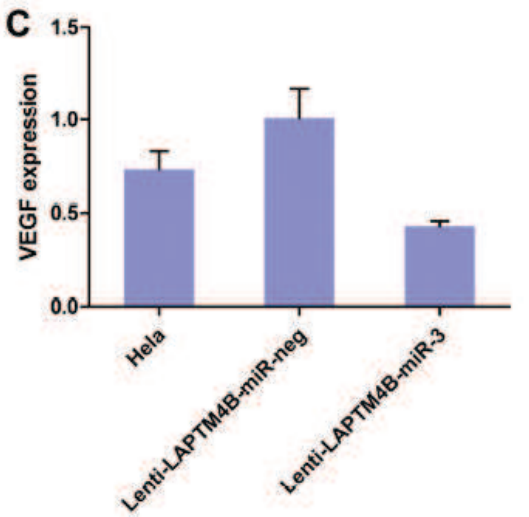

B

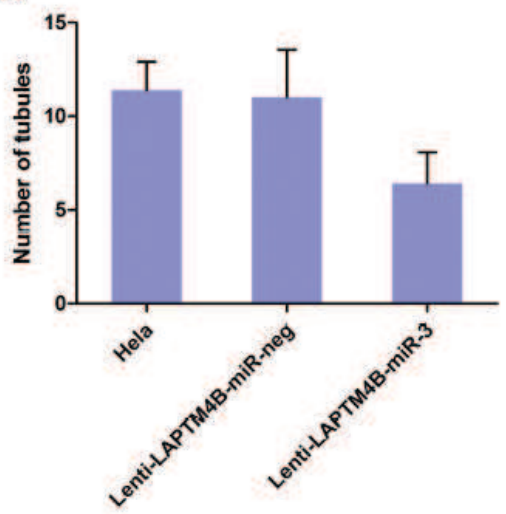

Fig. 7. LAPTM4B is involved in cervical cancer angiogenesis in vitro. (A and B) LAPTM4B blockade significantly suppressed tube formation by HUVECs. (C) ELISA assay results showing that LAPTM4B knockdown in HeLa cells inhibits the activities of VEGF.

Effects of LAPTM4B on the tube formation of endothelial cells

We assessed tube formation after LAPTM4B knockdown in HeLa cervical cancer cells. Tube numbers for each group were assessed under a light microscope. As expected, LAPTM4B blockade significantly suppressed tube formation by HUVECs (Fig. 7A, B). Taken together, these findings suggest that LAPTM4B expression in cervical cancer cells plays a pivotal role in promoting angiogenesis.

Detection of VEGF expression in the culture supernatant of tumor cell lines by ELISA

VEGF expression in the culture supernatants of tumor cell lines was detected for the three examined groups. Our results demonstrated that VEGF expression was significantly lower in the knockdown group compared with untreated HeLa cells (the control group) and lenti-LAPTM4B-miR-neg cells (the negative control group) (Fig. 7C). 


\section{Cellular Physiology Cell Physiol Biochem 2015;37:890-900 \\ \begin{tabular}{l|l|l} 
DOI: 10.1159/000430216 & O 2015 S. Karger AG, Basel
\end{tabular} \\ and Biochemistry Published online: September 18, $2015 \quad$ www.karger.com/cpb \\ Meng et al.: LAPTM4B Inhibits Invasion and Angiogenesis of Cervical Cancer}

\section{Discussion}

LAPTM4B was originally identified as an HCC-associated gene in the mammalian LAPTM family [17]. Studies have demonstrated that $L A P T M 4 B$ is up regulated in many human cancers and may play roles in the disease progression of malignant cells and in multidrug resistance $[18,19]$.Our previous research has identified LAPTM4B overexpression as an important prognostic marker of cervical carcinoma [16]. In addition, our prior findings have indicated that LAPTM4B gene polymorphisms may play an important role in genetic susceptibility to cervical cancer in the Chinese population [20]. However, the precise role of LAPTM4B in cervical cancer progression remains unknown.

To elucidate the biological functions of LAPTM4B in cervical cancer tumorigenesis, we chose to investigate these functions in HeLa cells through a loss-of-function study that involved an RNAi-mediated LAPTM4B knockdown. In this study, we have provided the first reports indicating that the silencing of LAPTM4B in HeLa cells significantly promoted tumor cell apoptosis and inhibited tumor growth, migration and invasion, resulting in the altered expression of certain metastasis- and invasion-related genes. More importantly, the RNAimediated knockdown of LAPTM4B reduced angiogenesis in tumors in a tube formation assay. Our data suggest that LAPTM4B is a potential therapeutic target for cervical carcinoma because the inhibition of LAPTM4B could produce significant antitumor and anti-angiogenic effects.

Previous studies have demonstrated that LAPTM4B overexpression activates certain proto-oncogenes and thereby promotes malignancy. The metastasis of tumor cells is dependent on the degradation of the components of the extracellular matrix by MMPs, particularly MMP-2 and MMP-9 [21]. Other studies have revealed the existence of latent links between LAPTM4B and MMPs [22]. Our study yielded similar results. We observed that LAPTM4B down regulation resulted in reduced expression and activity levels for MMP2 and MMP-9. Therefore, these results suggested a positive relationship between LAPTM4B and MMPs (specifically, MMP-2 and MMP-9).

Cyclin-dependent kinases (CDKs) participate in many cellular processes and play an important role in the regulation of the cell cycle and of transcription. Recently, CDK12 was identified as a key factor in cancer development that orchestrates the transcription of various genes, such as BRCA1, ATM, ATR, FANCI and FANCD2 [23]. Moreover, certain data indicate that for a variety of tumors, CDK12 has been identified as a factor that affects tumor sensitivity to cytostatics that promote DNA damage and the inhibition of DNA repair [23]. However, the precise contributions of CDK12 to tumor initiation and progression have not yet been fully elucidated [16]. In our study, LAPTM4B down regulation decreased CDK12 expression. These results indicated that LAPTM4B knockdown inhibits the aggressiveness of cervical cancer, possibly by regulating CDK12. Therefore, CDK12 involvement is implicated as an underlying mechanism of cancer development that is affected by an LAPTM4B blockade.

Angiogenesis is an important process in tumor progression [24, 25]. Hypoxia, which is a common characteristic of all rapidly growing solid malignancies, may trigger the activation of certain tumor-related signaling transduction pathways, such as the hypoxia-inducible factor$1 \alpha$ (HIF-1 $\alpha$ ) pathway [26]. HIF- $1 \alpha$ is a transcription factor known to be associated with most hypoxic solid tumors; this factor is consistently overexpressed in various human cancers and promotes tumorigenesis through angiogenesis $[27,28]$. One of the HIF-1-responsive genes is vascular endothelial growth factor (VEGF) [29]. VEGF and its family members are essential for the induction of tumor angiogenesis. The aberrant expression of VEGF is known to be a key regulator in hypoxia-induced angiogenesis [30]. Tumor growth relies on new blood vessels. Tang et al. have reported that the LAPTM4B protein is closely associated with progression and micro vessel density in the context of non-small cell lung cancer, suggesting that LAPTM4B could play an important role in tumor angiogenesis [10]. However, the exact mechanisms underlying this finding remain unclear. Based on our observations during this study, an LAPTM4B blockade may play a direct role in the degradation of HIF-1 $\alpha$ and the consequent suppression of subsequent VEGF signaling in cervical cancer cells, leading to 


\section{Cellular Physiology Cell Physiol Biochem 2015;37:890-900 \begin{tabular}{l|l|l}
\hline DOI: 10.1159/000430216 & O 2015 S. Karger AG, Basel
\end{tabular} and Biochemistry Published online: September 18, 2015 www.karger.com/cpb \\ Meng et al.: LAPTM4B Inhibits Invasion and Angiogenesis of Cervical Cancer}

the inhibition of hypoxia-induced tumor angiogenesis. We have provided the first report indicating that LAPTM4B suppresses angiogenesis through the HIF- $1 \alpha$ and VEGF pathway. In addition, we found that in HUVECs, an LAPTM4B blockade efficiently inhibited tube formation ability through the down regulation of VEGF expression; this result suggests that the LAPTM4B blockade induced abnormal angiogenesis through VEGF suppression. These results strongly suggest that LAPTM4B down regulation acts to inhibit tumor growth through the negative regulation of tumor angiogenesis mediated by the HIF1-VEGF pathway.

Our findings provide new evidence that LAPTM4B plays an important role in promoting tumor angiogenesis. Therefore, LAPTM4B may represent a novel therapeutic target in the development of cervical cancer treatments.

\section{Acknowledgments}

We express our thanks to Dr. Hongtao Song for the statistical analysis. This work was supported by grants of the National Natural Science Foundation of China (81201613), the Specialized Research Fund for the Doctoral Program of Higher Education (20122307120027), the Postdoctoral Foundation of Heilongjiang Province of China (LBH-Z11067), the scientific research project of Health Department of Heilongjiang Province (663) and the Haiyan Foundation of the Affiliated Tumor Hospital of Harbin Medical University / the Foundation of the Affiliated Tumor Hospital of Harbin Medical University (JJZ2011-04). The funders had no role in study design, data collection and analysis, decision to publish, or preparation of the manuscript.

\section{Disclosure Statement}

The authors declare that there are no conflicts of interest.

\section{References}

1 Siegel R, Ma J, Zou Z, Jemal A: Cancer statistics, 2014. CA Cancer J Clin 2014;64:9-29.

2 Ferlay J, Shin HR, Bray F, Forman D, Mathers C, Parkin DM: Estimates of worldwide burden of cancer in 2008: Globocan 2008. Int J Cancer 2010;127:2893-2917.

3 Li S, Hu T, Lv W, Zhou H, Li X, Yang R, Jia Y, Huang K, Chen Z, Wang S, Tang F, Zhang Q, Shen J, Zhou J, Xi L, Deng D, Wang H, Wang S, Xie X, Ma D: Changes in prevalence and clinical characteristics of cervical cancer in the people's republic of china: A study of 10,012 cases from a nationwide working group. Oncologist 2013;18:1101-1107.

4 Wei LH: Prevention and treatment of cervical cancer, it is a long-term and arduous task. Zhonghua Fu Chan Ke Za Zhi 2013;48:304-306.

5 Yee GP, de Souza P, Khachigian LM: Current and potential treatments for cervical cancer. Curr Cancer Drug Targets 2013;13:205-220.

6 Pectasides D, Kamposioras K, Papaxoinis G, Pectasides E: Chemotherapy for recurrent cervical cancer. Cancer Treat Rev 2008;34:603-613.

7 Tao X, Hu W, Ramirez PT, Kavanagh JJ: Chemotherapy for recurrent and metastatic cervical cancer. Gynecol Oncol 2008;110:S67-71.

8 Shao GZ, Zhou RL, Zhang QY, Zhang Y, Liu JJ, Rui JA, Wei X, Ye DX: Molecular cloning and characterization of laptm4b, a novel gene upregulated in hepatocellular carcinoma. Oncogene 2003;22:5060-5069.

9 Xiao M, Jia S, Wang H, Wang J, Huang Y, Li Z: Overexpression of laptm4b: An independent prognostic marker in breast cancer. J Cancer Res Clin Oncol 2013;139:661-667.

10 Tang H, Tian H, Yue W, Li L, Li S, Gao C, Si L, Qi L, Lu M: Overexpression of laptm4b is correlated with tumor angiogenesis and poor prognosis in non-small cell lung cancer. Med Oncol 2014;31:974. 


\section{Cellular Physiology Cell Physiol Biochem 2015;37:890-900 \begin{tabular}{l|l|l}
\hline DOI: 10.1159/000430216 & O 2015 S. Karger AG, Basel
\end{tabular} and Biochemistry Published online: September 18, 2015 www.karger.com/cpb \\ Meng et al.: LAPTM4B Inhibits Invasion and Angiogenesis of Cervical Cancer}

11 Zhang H, Tian B, Yu H, Yao H, Gao Z: Laptm4b-35 protein as a potential therapeutic target in gastric cancer. Tumour Biol 2014;35:12737-12742.

12 Yang H, Xiong F, Qi R, Liu Z, Lin M, Rui J, Su J, Zhou R: Laptm4b-35 is a novel prognostic factor of hepatocellular carcinoma. J Surg Oncol 2010;101:363-369.

13 Zhang H, Qi S, Zhang T, Wang A, Liu R, Guo J, Wang Y, Xu Y: Mir-188-5p inhibits tumour growth and metastasis in prostate cancer by repressing laptm4b expression. Oncotarget 2015;6:6092-6104.

14 Zhou L, He XD, Chen J, Cui QC, Qu Q, Rui JA, Zhao YP: Overexpression of laptm4b-35 closely correlated with clinicopathological features and post-resectional survival of gallbladder carcinoma. Eur J Cancer 2007;43:809-815.

15 Kasper G, Vogel A, Klaman I, Grone J, Petersen I, Weber B, Castanos-Velez E, Staub E, Mennerich D: The human laptm $4 \mathrm{~b}$ transcript is upregulated in various types of solid tumours and seems to play a dual functional role during tumour progression. Cancer Lett 2005;224:93-103.

16 Meng F, Luo C, Hu Y, Yin M, Lin M, Lou G, Zhou R: Overexpression of laptm4b-35 in cervical carcinoma: A clinicopathologic study. Int J Gynecol Pathol 2010;29:587-593.

17 Liu X, Zhou R, Zhang Q Zhang Y, Shao G, Jin Y, Zhang S, Lin M, Rui J, Ye D: [identification and characterization of laptm4b encoded by a human hepatocellular carcinoma-associated novel gene]. Beijing Da Xue Xue Bao 2003;35:340-347.

18 Li Y, Zhang Q, Tian R, Wang Q Zhao JJ, Iglehart JD, Wang ZC, Richardson AL: Lysosomal transmembrane protein laptm4b promotes autophagy and tolerance to metabolic stress in cancer cells. Cancer Res 2011;71:7481-7489.

19 Li L, Wei XH, Pan YP, Li HC, Yang H, He QH, Pang Y, Shan Y, Xiong FX, Shao GZ, Zhou RL: Laptm4b: A novel cancer-associated gene motivates multidrug resistance through efflux and activating pi3k/akt signaling. Oncogene 2010;29:5785-5795.

20 Meng F, Song H, Luo C, Yin M, Xu Y, Liu H, Zhou R, Lou G: Correlation of laptm4b polymorphisms with cervical carcinoma. Cancer 2011;117:2652-2658.

21 Yadav L, Puri N, Rastogi V, Satpute P, Ahmad R, Kaur G: Matrix metalloproteinases and cancer - roles in threat and therapy. Asian Pac J Cancer Prev 2014;15:1085-1091.

22 Zhou L, He XD, Yu JC, Zhou RL, Xiong FX, Qu Q, Rui JA: Expression of laptm4b in gallbladder carcinoma cells: The role in invasive potential. Hepatogastroenterology 2010;57:207-211.

23 Vrabel D, Svoboda M, Navratil J, Kohoutek J: Function of cdk12 in tumor initiation and progression and its clinical consequences. Klin Onkol 2014;27:340-346.

24 Zhu J, Sheng J, Dong H, Kang L, Ang J, Xu Z: Phospholipid scramblase 1 functionally interacts with angiogenin and regulates angiogenin-enhanced rrna transcription. Cell Physiol Biochem 2013;32:16951706.

25 Zhu X, Er K, Mao C, Yan Q Xu H, Zhang Y, Zhu J, Cui F, Zhao W, Shi H: Mir-203 suppresses tumor growth and angiogenesis by targeting vegfa in cervical cancer. Cell Physiol Biochem 2013;32:64-73.

26 Kimura H, Weisz A, Ogura T, Hitomi Y, Kurashima Y, Hashimoto K, D'Acquisto F, Makuuchi M, Esumi H: Identification of hypoxia-inducible factor 1 ancillary sequence and its function in vascular endothelial growth factor gene induction by hypoxia and nitric oxide. J Biol Chem 2001;276:2292-2298.

27 Semenza GL: Hif-1 and tumor progression: Pathophysiology and therapeutics. Trends Mol Med 2002;8:S6267.

28 Semenza GL: Hif-1: Using two hands to flip the angiogenic switch. Cancer Metastasis Rev 2000;19:59-65.

29 Stacker SA, Caesar C, Baldwin ME, Thornton GE, Williams RA, Prevo R, Jackson DG, Nishikawa S, Kubo H, Achen MG: Vegf-d promotes the metastatic spread of tumor cells via the lymphatics. Nat Med 2001;7:186191.

30 Goel HL, Mercurio AM: Vegf targets the tumour cell. Nat Rev Cancer 2013;13:871-882. 